\title{
Split-Order Distance for Clustering and Classification Hierarchies
}

\author{
Qi Zhang, Eric Yi Liu, Abhishek Sarkar, and Wei Wang \\ Department of Computer Science \\ University of North Carolina at Chapel Hill
}

\begin{abstract}
Clustering and classification hierarchies are organizational structures of a set of objects. Multiple hierarchies may be derived over the same set of objects, which makes distance computation between hierarchies an important task for summarization and similarity search of hierarchical patterns. In this paper, we model the classification and clustering hierarchies as rooted, leaf-labeled, unordered trees. We propose a novel distance metric Split-Order distance to evaluate the organizational structure difference between two hierarchies over the same set of leaf objects. The Split-Order distance reflects the order in which subsets of the tree leaves are differentiated from each other and can be used to explain the relationships between the leaf objects. We also propose an efficient algorithm for computing Split-Order distance between two trees in $O\left(n^{2} d^{4}\right)$ time, where $n$ is the number of leaves, and $d$ is the maximum number of children of any node. Our experiments on both real and synthetic data demonstrate the efficiency and effectiveness of our algorithm.
\end{abstract}

Key words: ENTER KEYWORDS HERE

\section{Introduction}

Clustering and classification hierarchies are important tools for capturing the relationships among objects. Two representative examples are dendrograms illustrating the hierarchical clustering result (Fig. 1(a)) and taxonomies classifying the biological species (Fig. 1(b)). A hierarchy organizes the set of objects in a tree, where objects with higher similarity are grouped together at a lower level, before objects that are more distant merge into bigger groups. The groups at the intermediate levels represent subclusters or subclasses.

Clustering and classification hierarchies can be automatically constructed given the pair-wise distance matrix over the set of objects. However, different models and methods may yield different estimates for the hierarchical structure. Quantifying the differences between hierarchies becomes crucial for tasks such as summarization of hierarchical patterns, computation of consensus hierarchies, and comparison of hierarchically structured data. A motivating example is the comparison of phylogenetic taxomonies, or phylogeny trees. A phylogeny tree (Fig. 1(b)) describes the evolutionary relationship between different organisms. 


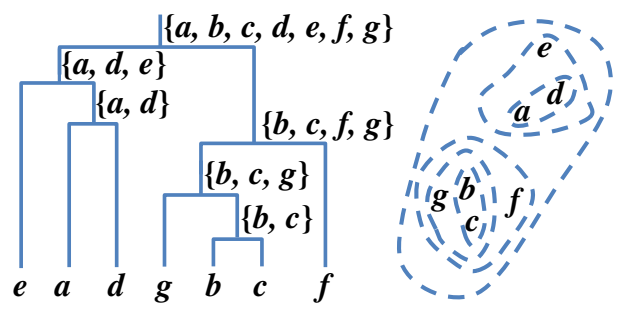

(a) A dendrogram over 7 objects

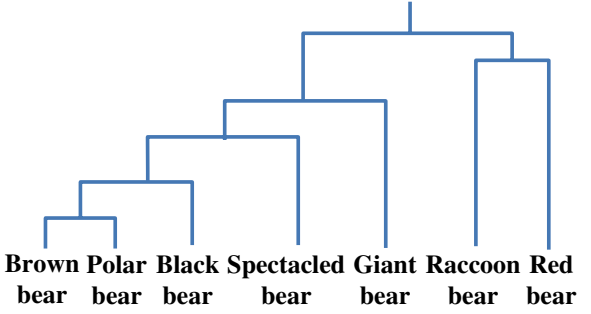

(b) A taxnomony over 7 species

Fig. 1. Example clustering and classification hierarchies.

The leaves of the tree represent the species, and the internal nodes correspond to the specialization events, where the evolution diverge in different directions to generate subspecies. The root of the tree is the most recent common ancestor for all the species. Different hierarchies can be derived for a given set of species using different phylogeny tree construction algorithms, such as UPGMA [Sneath73], neighbor-join [Saitou87], maximum parsimony [Felsenstein78], etc. Comparing the similarity of different phylogeny trees is important for evaluating different algorithms and deriving the consensus phylogeny tree.

Many algorithms have been proposed for comparing general tree topologies. Tree edit distance is a classic metric to compare two trees with both internal nodes and leaf nodes labeled. As opposed to a general tree structure, clustering and classification hierarchies are leaf-labeled trees; leaves represent the objects. The tree edit distance computation for unordered trees is NP-complete [Zhang89]. Polynomial-time algorithms [Zhang89,Demaine07,Touzet03,Bille05,Klein98,Lu79,Wang94] only exist for ordered trees. As for classification or clustering hierarchies, there is no specific order among siblings. If we were to consider classification or clustering hierarchies as ordered, fully-labeled trees, metrics such as the tree edit distance may produce results which do not agree with our understanding of the hierarchies. Consider four trees presented in Fig. 2. $T_{1}, T_{2}, T_{3}$, and $T_{4}$ are different hierarchies over a common object set $\{a, b, c, d\}$. To apply the ordered tree edit distance, we also impose labels for the internal nodes. As classification hierarchies, $T_{2}$ and $T_{4}$ are the same as $T_{1}$. The objects are classified in the same way in each of the trees; specifically, the order in which the objects are differentiated from each other is the same. However, the tree edit distance between $T_{1}$ and $T_{2}$ is 2 since leaves $a$ and $b$ are transposed. The tree edit distance between $T_{1}$ and $T_{4}$ is also 2 since two internal nodes are transposed. In addition, $T_{3}$ has a tree edit distance of 2 from $T_{1}$, which implies that $T_{1}, T_{2}$, and $T_{3}$ are equidistant from $T_{1}$. However, the way in which $a$ and $d$ are classified in $T_{3}$ is very different from the way they are classified in $T_{1}$. On the other hand, $a$ and $d$ are classified in the same way in both $T_{1}$ and $T_{2}$.

In this paper, we propose a novel distance metric, Split-Order distance, for comparing clustering/classification hierarchies. Different from a general tree 


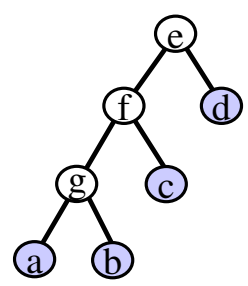

(a) $T_{1}$

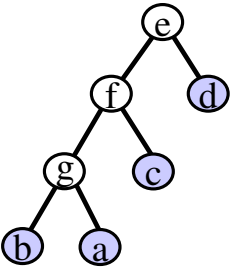

(b) $T_{2}$

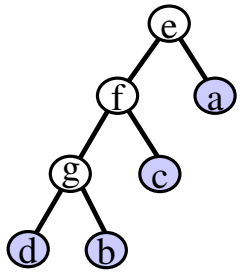

(c) $T_{3}$

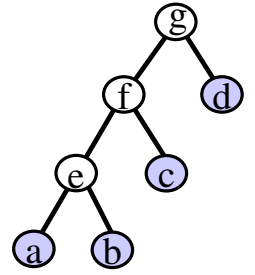

(d) $T_{4}$

Fig. 2. An example with four trees. $T_{1}, T_{2}, T_{3}$, and $T_{4}$ are four hierarchies with the same set of objects(leaf labels) $\{a, b, c, d\}$. To apply the tree edit distance, we also impose labels for internal nodes. Trees are considered as ordered.

structure, a clustering/classification hierarchy can be modeled as rooted, unordered, leaf-labeled trees. Essential to these hierarchical structures is the set of relationship among the leaf objects captured by the hierarchy: an object is more closely related to another object which it merges with at a lower level than another object which it merges with at a higher level. We refer to a split between two objects as the smallest subcluster/subclass in the hierarchy where both objects belong to. A split corresponds to an internal node in the tree which is the most recent common ancestor of the two leaf nodes representing the two objects. All the splits form a partial order which uniquely determines the relationship among all the objects captured by the hierarchy. For example, in Fig. 1(a), the split between $\mathrm{O}_{2}$ and $\mathrm{O}_{3}$ happens at a lower level than the split between $\mathrm{O}_{3}$ and $O_{6}$ does; in Fig. 1(b), the split between polar bear and red bear occurs at a higher level than the split between polar bear and brown bear. We define the Split-Order distance between two hierarchical structures based on the order of the splits occuring in the tree. Our contributions can be summarized as follows:

1. We define a novel distance metric, Split-Order distance, between two clustering or classification hierarchies. We prove that Split-Order distance is a metric.

2. We prove that a complete set of split orders uniquely defines a hierarchical structure. In addition, we propose an algorithm for reconstructing the hierarchy using a set of split orders.

3. We propose an efficient algorithm for computing the Split-Order distance between any two hierarchies. Our algorithm takes $O\left(n^{2} d^{4}\right)$ time, where $n$ is the number of leaves, $d$ is the maximum degree of any node.

The rest of the paper is organized as follows. We review the related work in Section 2 and introduce the preliminaries in Section 3. In Section 4, we discuss the formal definition of Split-Order distance. We present our algorithm for efficient computation of the Split-Order distance in Section 5. The experimental results are reported in Section 6, and Section 7 concludes the paper. 


\section{Related Work}

Many algorithms have been proposed for comparing tree-like or hierarchically structured data. One of the tree distance metrics which has been extensively studied is the tree edit distance. The tree edit distance evaluates the cost of transforming a tree into another tree through a sequence of operations, such as deleting, inserting, and relabeling nodes. A cost is defined for each operation, and the minimum of the total cost of all operations in a sequence is the tree edit distance. Among the different tree edit distance computation algorithms [Demaine07,Touzet03,Bille05,Klein98,Lu79,Wang94,Zhang89], two representative ones are Zhang-Shasha [Zhang89] and Klein [Klein98]. Both algorithms use dynamic programming techniques with worst-case complexities $O\left(n^{4}\right)$ and $O\left(n^{3} \log n\right)$, respectively. Besides tree edit distance, another type of tree distance metrics compare the trees based on the structures they share such as maximum agreement subtrees [Amir97], cousin-pairs [Shasha04], etc. However, as mentioned in the previous section, these general tree distance metrics might not be readily used for comparing clustering or classification hierarchies which are usually modeled as rooted, unordered, leaf-labeled trees, and are able to capture the relationship among leaf objects. For phylogeny trees, several distance metrics have been proposed incorporating biologically meaningful definitions [Wang03,Robinson81,Robinson79,Allen01,Estabrook85]. But they may not work well on general clustering/classification hierarchies.

\section{Preliminaries}

We model the clustering and classification hierarchies as rooted, unordered, leaflabeled trees, as shown in Fig. 3 .

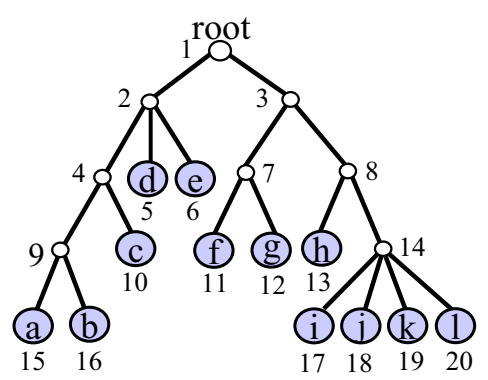

Fig. 3. A rooted, unordered, leaf-labeled tree representing a clustering or classification hierarchy over a set of objects $\Sigma=\{a, b, c, d, e, f, g, h, i, j, k, l\}$.

The leaf nodes represent the objects. Each internal node represents the split of a cluster/class into several subclusters/subclasses. The root represents the entire set of objects. We consider unordered trees, since the sibling order does not exist 
in classification/clustering hierarchies. We also assume that each internal node has at least two children; each child represents a different subclass/subcluster resulting from the split.

Let the set of leaf labels be $\Sigma=\left\{\sigma_{1}, \ldots, \sigma_{n}\right\}$. We refer to a leaf node using its label in the following discussion. All nodes in the tree are uniquely numbered for easy reference (see Fig. 3). For a leaf node $\sigma_{i} \in \Sigma$, the internal nodes on the path from $\sigma_{i}$ to the root are ancestors of $\sigma_{i}$. The ancestors represent the subclusters/subclasses containing $\sigma_{i}$. For any two leaf nodes $\sigma_{i}, \sigma_{j}$, we define the Split between $\sigma_{i}, \sigma_{j}$ as follows:

Definition 1. The Split between $\sigma_{i}, \sigma_{j}$ (denoted as Split $\left(\sigma_{i}, \sigma_{j}\right)$ ). Given a rooted, unordered tree $T$ with a set of leaf labels $\Sigma$, for any two leaf nodes $\sigma_{i}, \sigma_{j} \in \Sigma$, the Split between $\sigma_{i}, \sigma_{j}$ is defined as the most recent common ancestor of $\sigma_{i}$ and $\sigma_{j}$ in $T$.

$\operatorname{Split}\left(\sigma_{i}, \sigma_{j}\right)$ represents the smallest cluster/class which includes both $\sigma_{i}$ and $\sigma_{j}$, i.e., the split during the top-down hierarchical clustering/classification process which divides $\sigma_{i}, \sigma_{j}$ into different subclusters/classes. For example, in Fig. 3 , nodes $1,2,4$, and 9 are ancestors of leaf node $a$, nodes 1,2 , and 4 are ancestors of leaf node $c$. Therefore, $\operatorname{Split}(a, c)$ is node 4 .

In the following section, we define the Split-Order distance for any two hierarchies $T_{1}, T_{2}$ over the same set of leaf objects $\Sigma$.

\section{Split-Order Distance}

Given a rooted, unordered tree $T$ with a set of leaf labels $\Sigma$, for any leaf node $\sigma_{i}$, the order of the splits which separate $\sigma_{i}$ and the remaining leaf nodes determines the relationship between $\sigma_{i}$ and other leaves. By "order", we mean that the split happens "earlier" (closer to the root) or "later" (closer to the leaf $\sigma_{i}$ ). Formally, for any leaf node $\sigma_{i}$, we define the order relationship of any two splits $\operatorname{Split}\left(\sigma_{i}, \sigma_{j}\right), \operatorname{Split}\left(\sigma_{i}, \sigma_{k}\right)$ as follows:

Definition 2. Split-Order Relations. Given a rooted, unordered tree with a set of leaf labels $\Sigma$, for any leaf node $\sigma_{i} \in \Sigma$, and any two other leaf nodes $\sigma_{j}$, $\sigma_{k} \in \Sigma$, we define

1. SplitOrder $\left(\sigma_{i}, \sigma_{j}, \sigma_{k}\right)=$ 'ঝ', if $\operatorname{Split}\left(\sigma_{i}, \sigma_{j}\right)$ is an ancestor of $\operatorname{Split}\left(\sigma_{i}, \sigma_{k}\right)$, $\left(\operatorname{Split}\left(\sigma_{i}, \sigma_{j}\right)\right.$ happens earlier than $\left.\operatorname{Split}\left(\sigma_{i}, \sigma_{k}\right)\right)$;

2. SplitOrder $\left(\sigma_{i}, \sigma_{j}, \sigma_{k}\right)={ }^{\prime} \succ$ ', if $\operatorname{Split}\left(\sigma_{i}, \sigma_{k}\right)$ is an ancestor of $\operatorname{Split}\left(\sigma_{i}, \sigma_{j}\right)$, $\left(\operatorname{Split}\left(\sigma_{i}, \sigma_{j}\right)\right.$ happens later than $\left.\operatorname{Split}\left(\sigma_{i}, \sigma_{k}\right)\right)$;

3. SplitOrder $\left(\sigma_{i}, \sigma_{j}, \sigma_{k}\right)='=$ ', if $\operatorname{Split}\left(\sigma_{i}, \sigma_{j}\right)=\operatorname{Split}\left(\sigma_{i}, \sigma_{k}\right),\left(\operatorname{Split}\left(\sigma_{i}, \sigma_{j}\right)\right.$ happens at the same time with $\left.\operatorname{Split}\left(\sigma_{i}, \sigma_{k}\right)\right)$.

For example, in Fig. 3, we have SplitOrder $(a, b, c)={ }^{`} \succ ', \operatorname{SplitOrder}(a, g, d)=$ 'ঝ', and SplitOrder $(a, f, h)=$ '='. The topology of $T$ determines a map $\Sigma \times \Sigma \times \Sigma \mapsto$ $\{\prec, \succ,=\}$. In the following discussion, we show that the complete set of the Split-Order relations $\Theta=\left\{\operatorname{SplitOrder}\left(\sigma_{i}, \sigma_{j}, \sigma_{k}\right)=\theta_{i, j, k}\right\}, \forall \sigma_{i}, \sigma_{j}, \sigma_{k} \in \Sigma$, $\theta_{i, j, k} \in\{\prec, \succ,=\}$ can serve as a unique signature of $T$. 
Theorem 1. Given a complete set of the Split-Order relations $\Theta=\left\{\operatorname{SplitOrder}\left(\sigma_{i}, \sigma_{j}, \sigma_{k}\right)=\right.$ $\left.\theta_{i, j, k}\right\}, \forall \sigma_{i}, \sigma_{j}, \sigma_{k} \in \Sigma, \theta_{i, j, k} \in\{\prec, \succ,=\}$, we can either reconstruct a unique tree or declare that a tree corresponding to $\Theta$ does not exist.

Proof. We prove the theorem by describing the following recursive algorithm to reconstruct a unique tree or report the nonexistence of a tree.

In the beginning, we start with the set of leaf nodes $\Sigma$. All the nodes are unprocessed initially. Each time we pick an unprocessed leave node from $\Sigma$ for processing until all nodes in $\Sigma$ are processed. Let $\sigma_{i}$ be the node we pick. We find the set of leaf nodes $\left\{\sigma_{j}\right\}$ which have the latest split with $\sigma_{i}$ :

$$
\left\{\sigma_{j} \mid \neg \exists \sigma_{k} \in \Sigma, \sigma_{k} \neq \sigma_{i}, \operatorname{SplitOrder}\left(\sigma_{i}, \sigma_{j}, \sigma_{k}\right)=^{\prime} \prec^{\prime}\right\}
$$

It is intuitive to prove that $\sigma_{i}$ and all nodes in $\left\{\sigma_{j}\right\}$ must be siblings. Next, we examine whether there are any conflicting Split-Order relations in $\Theta$. For any two leaf nodes $\sigma_{j_{1}}, \sigma_{j_{2}} \in\left\{\sigma_{j}\right\}$, we check whether the following two equations hold:

$$
\begin{aligned}
& \text { SplitOrder }\left(\sigma_{i}, \sigma_{j_{1}}, \sigma_{k}\right)=\operatorname{SplitOrder}\left(\sigma_{i}, \sigma_{j_{2}}, \sigma_{k}\right) \\
& \forall \sigma_{k} \in \Sigma
\end{aligned}
$$

$$
\begin{aligned}
& \text { SplitOrder }\left(\sigma_{j_{1}}, \sigma_{k}, \sigma_{l}\right)=\operatorname{SplitOrder}\left(\sigma_{j_{2}}, \sigma_{k}, \sigma_{l}\right)=\operatorname{SplitOrder}\left(\sigma_{i}, \sigma_{k}, \sigma_{l}\right) \\
& \forall \sigma_{k}, \sigma_{l} \in \Sigma
\end{aligned}
$$

If any of the above equations does not hold, which implies conflicting SplitOrder relations in $\Sigma$, a tree conforming to $\Sigma$ does not exist. Due to limited space, we omit the proof here. If both equations hold, we create a parent node $\sigma_{\text {new }}$ for $\sigma_{i}$ and all leaf nodes in $\left\{\sigma_{j}\right\}$. We add $\sigma_{\text {new }}$ to $\Sigma^{(1)}$, and mark $\left\{\sigma_{j}\right\}$ and $\sigma_{i}$ as processed.

If $\Sigma$ still contains unprocessed nodes, we pick another leaf node, and start the process again. After all nodes in $\Sigma$ have been processed, we start the next iteration with $\Sigma^{(1)}$. All nodes in $\Sigma^{(1)}$ will be treated as leaf nodes, and their Split-Order relations are determined by the nodes they represent, as follows:

$$
\operatorname{SplitOrder}\left(\sigma_{u^{\prime}}^{(1)}, \sigma_{v^{\prime}}^{(1)}, \sigma_{w^{\prime}}^{(1)}\right)=\operatorname{SplitOrder}\left(\sigma_{u}, \sigma_{v}, \sigma_{w}\right)
$$

where $\sigma_{u}$ is any child of $\sigma_{u^{\prime}}^{(1)}, \sigma_{v}$ is any child of $\sigma_{v^{\prime}}^{(1)}$, and $\sigma_{w}$ is any child of $\sigma_{w^{\prime}}^{(1)}$.

We recurse until at iteration $p, \Sigma^{(p)}$ contains only the root node. If at any point, either Equation 2 or 3 does not hold, the process aborts and reports the nonexistence of a tree. The complete algorithm is shown in Algorithm 1.

Definition 3. Split-Order distance. For two rooted, unordered trees $T, T^{\prime}$ with the same set of leaf labels $\Sigma$, and their corresponding Split-Order relationship sets $\Theta, \Theta^{\prime}$, the Split-Order distance between $T$ and $T^{\prime}$ is defined as

$$
\begin{aligned}
& \operatorname{SODist}\left(T, T^{\prime}\right)= \\
& \mid\left\{\operatorname{SplitOrder}\left(\sigma_{i}, \sigma_{j}, \sigma_{k}\right) \text { s.t. SplitOrder }\left(\sigma_{i}, \sigma_{j}, \sigma_{k}\right) \neq \operatorname{SplitOrder}\left(\sigma_{i}^{\prime}, \sigma_{j}^{\prime}, \sigma_{k}^{\prime}\right)\right\} \mid
\end{aligned}
$$




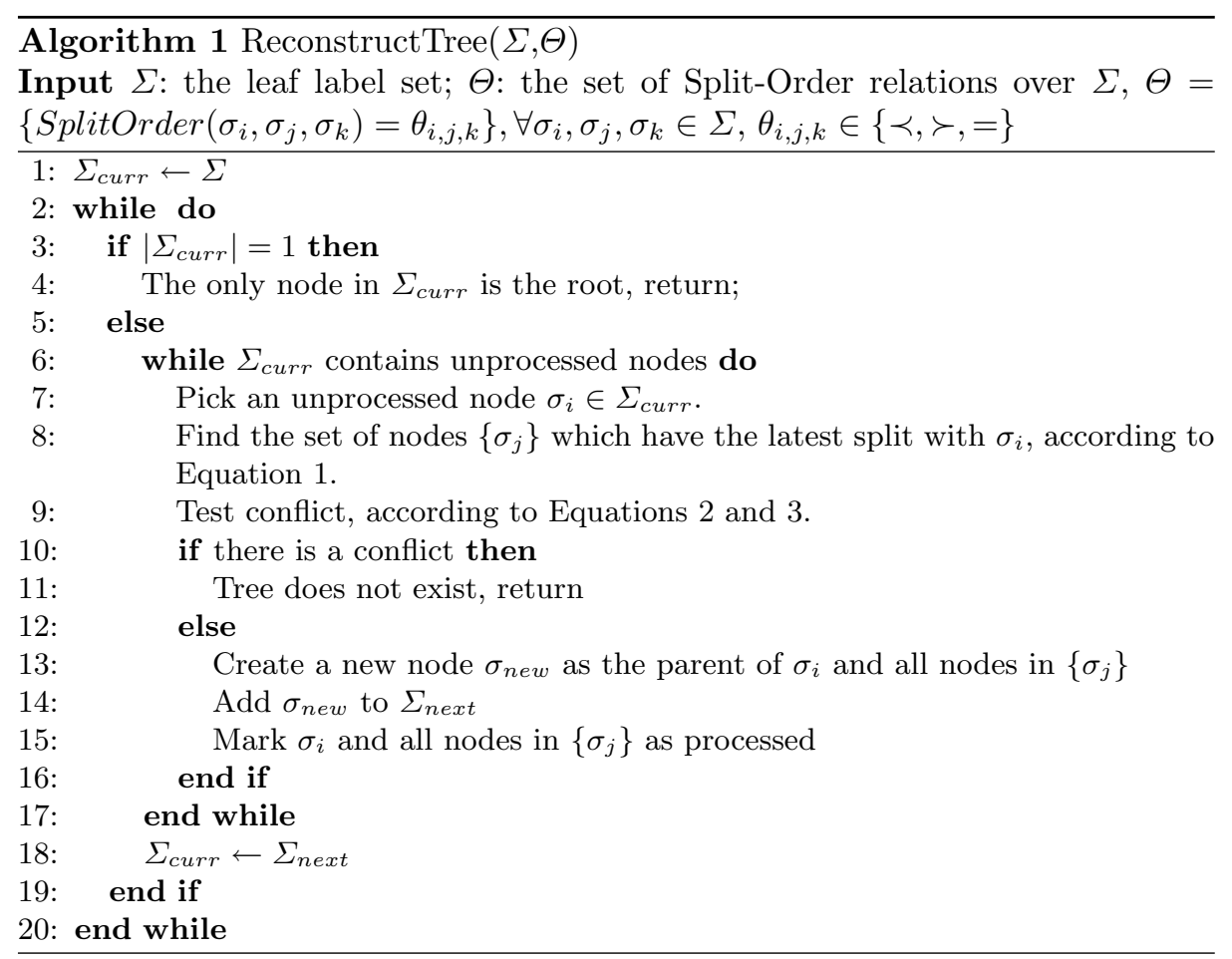

where $\left(\operatorname{SplitOrder}\left(\sigma_{i}, \sigma_{j}, \sigma_{k}\right)=\theta_{i, j, k}\right) \in \Theta$, and $\left(\operatorname{SplitOrder}\left(\sigma_{i}^{\prime}, \sigma_{j}^{\prime}, \sigma_{k}^{\prime}\right)=\theta_{i, j, k}^{\prime}\right) \in$ $\Theta^{\prime}$.

The relative Split-Order distance is defined as:

$$
\operatorname{SODistRel}\left(T, T^{\prime}\right)=\operatorname{SODist}\left(T, T^{\prime}\right) / n^{3}
$$

where $n=|\Sigma|$. In other words, $n^{3}=|\Theta|=\left|\Theta^{\prime}\right|$. Note that SODistRel $(T$, $\left.T^{\prime}\right) \in[0,1]$.

Theorem 2. The Split-Order distance is a metric.

Proof. Assume that we have three rooted, unordered trees $T_{1}, T_{2}, T_{3}$ with the same set of leaf labels $\Sigma$. The proof for symmetry, identity and non-negativity properties are intuitive and omitted here. We will prove the triangle inequality: $\operatorname{SODist}\left(T_{1}, T_{2}\right) \leq \operatorname{SODist}\left(T_{1}, T_{3}\right)+\operatorname{SODist}\left(T_{2}, T_{3}\right)$. Denote the total number of Split-Order relations for each tree as $m$. Then the number of common Split-Order relations shared between $T_{1}$ and $T_{3}$ is $m-\operatorname{SODist}\left(T_{1}, T_{3}\right)$, and the number of common Split-Order relations shared between $T_{2}$ and $T_{3}$ is $m-\operatorname{SODist}\left(T_{2}, T_{3}\right)$. Therefore, the number of common Split-Order relations shared between $T_{1}, T_{2}$, and $T_{3}$ is at least

$$
\begin{aligned}
& \left(m-\operatorname{SODist}\left(T_{1}, T_{3}\right)\right)+\left(m-\operatorname{SOD} \operatorname{Dist}\left(T_{2}, T_{3}\right)\right)-m \\
& =m-\left(\operatorname{SODist}\left(T_{1}, T_{3}\right)+\operatorname{SODist}\left(T_{2}, T_{3}\right)\right)
\end{aligned}
$$


Thus, the number of Split-Order relations which are different between $T_{1}$ and $T_{2}$ is at most

$$
\begin{aligned}
& m-\left(m-\left(S O D i s t\left(T_{1}, T_{3}\right)+\operatorname{SODist}\left(T_{2}, T_{3}\right)\right)\right) \\
& =\operatorname{SODist}\left(T_{1}, T_{3}\right)+\operatorname{SODist}\left(T_{2}, T_{3}\right)
\end{aligned}
$$

\section{Split-Order Distance Computation}

We propose an efficient algorithm for computing the Split-Order distance between any two trees $T_{1}, T_{2}$ over the same set of leaf objects $\Sigma$.

The naive algorithm computes the complete set of the Split-Order relations for both trees and counts the number of different Split-Order relations in two trees. Since there are $O\left(n^{3}\right)$ Split-Order relations for each tree, counting the different Split-Order relations alone will take $O\left(n^{3}\right)$ time. In fact, computing a single Split-Order relation has more than $O(1)$ complexity. In the following discussion, we propose an efficient algorithm for computing Split-Order distance which takes only $O\left(n^{2} d^{4}\right)$ time, where $d$ is the maximum degree of any node.

Let $\Theta(T)$ denote the complete set of the Split-Order relations of tree $T$. For each internal node $n_{p}$, we compute a subset of $\Theta(T)$, denoted as $\operatorname{SplitOrderSet}\left(T, n_{p}\right)$ :

$$
\begin{aligned}
& \text { SplitOrderSet }\left(T, n_{p}\right)=\left\{\text { SplitOrder }\left(\sigma_{i_{1}}, \sigma_{i_{2}}, \sigma_{i_{3}}\right)=\theta_{i_{1}, i_{2}, i_{3}}\right. \text { where } \\
& \left.\left(\text { Split }\left(\sigma_{i_{1}}, \sigma_{i_{2}}\right)=n_{p}\right) \wedge\left(\left(\text { SplitOrder }\left(\sigma_{i_{1}}, \sigma_{i_{2}}, \sigma_{i_{3}}\right)=\theta_{i_{1}, i_{2}, i_{3}}\right) \in \Theta(T)\right)\right\}
\end{aligned}
$$

It is easy to prove that $\Theta(T)$ can be divided into disjoint sets of $\operatorname{SplitOrderSet}\left(T, n_{p}\right)$ :

$$
\begin{gathered}
\cup_{n_{p}} \operatorname{SplitOrderSet}\left(T, n_{p}\right)=\Theta(T) \\
\operatorname{SplitOrderSet}\left(T, n_{p_{1}}\right) \cap \operatorname{SplitOrderSet}\left(T, n_{p_{2}}\right)=\phi
\end{gathered}
$$

The basic idea of our Split-Order distance computation algorithm is to count, for any internal node $n_{p}$ in $T$ and any internal node $n_{p^{\prime}}$ in $T^{\prime}$, the number of common Split-Order relations associated with them, i.e., $\mid \operatorname{SplitOrderSet}\left(T, n_{p}\right) \cap$ SplitOrderSet $\left(T^{\prime}, n_{p^{\prime}}\right) \mid$. In the following discussion, we only consider the SplitOrder relations of type ' $\succ$ ' and ' $=$ ', since we have

$$
\begin{aligned}
& \text { SplitOrder }\left(\sigma_{i_{1}}, \sigma_{i_{2}}, \sigma_{i_{3}}\right)==^{\prime} \prec^{\prime} \Leftrightarrow \\
& \operatorname{SplitOrder}\left(\sigma_{i_{1}}, \sigma_{i_{3}}, \sigma_{i_{2}}\right)={ }^{\prime} \succ^{\prime}
\end{aligned}
$$

The total number of common Split-Order relations is twice the number of total common ' $\succ$ '-type Split-Order relations plus the number of total common '='type Split-Order relations. Furthermore, we only consider Split-Order relations where $\sigma_{i_{1}}, \sigma_{i_{2}}$, and $\sigma_{i_{3}}$ are three different leaf labels. If any two of them are the same we already know that the relation is common between $\Theta(T)$ and $\Theta\left(T^{\prime}\right)$.

We first explain how to compute the Split-Order relations of type ' $\succ$ ' and ' $=$ ' in $\operatorname{SplitOrderSet}\left(T, n_{p}\right)$ given a tree $T$ and an internal node $n_{p}$. Denote the set of leaf nodes which are inside the subtree rooted at $n_{p}$ as Leaves $\left(n_{p}\right)$, and the $k^{\text {th }}$ child of $n_{p}$ as $\operatorname{Child}\left(n_{p}, k\right)$ (see Fig. 4). It is easy to prove that Leaves $\left(n_{p}\right)=$ 
$\cup_{k}$ Leaves $\left(\operatorname{Child}\left(n_{p}, k\right)\right)$, and Leaves $\left(\operatorname{Child}\left(n_{p}, k\right)\right) \cap \operatorname{Leaves}\left(\operatorname{Child}\left(n_{p}, k^{\prime}\right)\right)=$ $\phi$. Here we assume that all child nodes are in an ordered list for the convenience of discussion. The child nodes can be of any order. For example in Fig. 3, consider $n_{4}$, Leaves $\left(n_{4}\right)=\{a, b, c\} . n_{9}$ and $n_{10}$ are the children of $n_{4}$, and Leaves $\left(n_{9}\right)=\{a, b\}$, Leaves $\left(n_{10}\right)=\{c\}$. Now we determine the necessary conditions for SplitOrder $\left(\sigma_{i_{1}}, \sigma_{i_{2}}, \sigma_{i_{3}}\right)$ to be of ' $\succ$ ' or '=' type. If $\sigma_{i_{1}} \in$ Leaves $\left(\operatorname{Child}\left(n_{p}, k_{1}\right)\right)$, and $\sigma_{i_{2}} \in \operatorname{Leaves}\left(\operatorname{Child}\left(n_{p}, k_{2}\right)\right)$, we know that $k_{1} \neq k_{2}$. Otherwise, $\operatorname{Split}\left(\sigma_{i_{1}}, \sigma_{i_{2}}\right)$ is $\operatorname{Child}\left(n_{p}, k_{1}\right)$ or a descendent of $\operatorname{Child}\left(n_{p}, k_{1}\right)$, but not $n_{p}$. Therefore, $\sigma_{i_{1}}, \sigma_{i_{2}}$ are from different child nodes of $n_{p}$. For the requirement of $\sigma_{i_{3}}$, we have $\sigma_{i_{3}} \in \Sigma \backslash$ Leaves $\left(\operatorname{Child}\left(n_{p}, k_{1}\right)\right)$. Otherwise, if $\sigma_{i_{3}} \in$ Leaves $\left(\operatorname{Child}\left(n_{p}, k_{1}\right)\right)$, we have $\operatorname{Split}\left(\sigma_{i_{1}}, \sigma_{i_{2}}, \sigma_{i_{3}}\right)={ }^{\prime} \prec$ '. Furthermore, depending on the type of the Split-Order relationship (' $\succ$ ' or ' $=$ '), $\sigma_{i_{3}}$ should belong to either $\Sigma \backslash$ Leaves $\left(n_{p}\right)$ or Leaves $\left(n_{p}\right) \backslash$ Leaves $\left(\operatorname{Child}\left(n_{p}, k_{1}\right)\right)$. In summary, $\sigma_{i_{1}}$, $\sigma_{i_{2}}, \sigma_{i_{3}}$ in a ' $\succ$ '-type Split-Order relation in $\operatorname{SplitOrderSet}\left(T, n_{p}\right)$ should satisfy (see Fig. 4):

$$
\left\{\begin{array}{l}
\sigma_{i_{1}} \in \operatorname{Leaves}\left(\operatorname{Child}\left(n_{p}, k_{1}\right)\right) \\
\sigma_{i_{2}} \in \operatorname{Leaves}\left(\operatorname{Child}\left(n_{p}, k_{2}\right)\right) \wedge k_{1} \neq k_{2} \\
\sigma_{i_{3}} \in \Sigma \backslash \operatorname{Leaves}\left(n_{p}\right)
\end{array}\right.
$$

where $1 \leq k_{1}, k_{2} \leq K$, and $K$ is the number of children of node $n_{p} . \sigma_{i_{1}}, \sigma_{i_{2}}, \sigma_{i_{3}}$ in an '='-type SplitOrder relation in $\operatorname{SplitOrderSet}\left(T, n_{p}\right)$ should satisfy (see Fig. 4):

$$
\left\{\begin{array}{l}
\sigma_{i_{1}} \in \operatorname{Leaves}\left(\operatorname{Child}\left(n_{p}, k_{1}\right)\right) \\
\sigma_{i_{2}} \in \operatorname{Leaves}\left(\operatorname{Child}\left(n_{p}, k_{2}\right)\right) \wedge k_{1} \neq k_{2} \\
\sigma_{i_{3}} \in \operatorname{Leaves}\left(\operatorname{Child}\left(n_{p}, k_{3}\right)\right) \wedge k_{1} \neq k_{3} \wedge \sigma_{i_{2}} \neq \sigma_{i_{3}}
\end{array}\right.
$$

where $1 \leq k_{1}, k_{2} \leq K$, and $K$ is the number of children of $n_{p}$. As an example, for node $n_{7}$ in Fig. 3, we have SplitOrder $(f, h, i)={ }^{\prime}=$ ' and $\operatorname{SplitOrder}(f, h, a)={ }^{\prime} \succ$ '.

Now we explain how to compute the size of SplitOrderSet $\left(T, n_{p}\right) \cap S p l i t O r d e r S e t$ $\left(T^{\prime}, n_{p^{\prime}}\right)$ for a pair of internal nodes $n_{p}, n_{p^{\prime}}$ in tree $T$ and $T^{\prime}$, respectively. As discussed earlier, we consider the ' $\succ$ ' and ' $=$ ' Split-Order relations. The number of common ' $\succ$ ' Split-Order relations Shared $_{\succ}\left(n_{p}, n_{p^{\prime}}\right)$ can be computed as follows:

$$
\begin{aligned}
& \text { Shared } \succ\left(n_{p}, n_{p^{\prime}}\right)=\Sigma_{1 \leq k_{1} \neq k_{2} \leq K, 1 \leq k_{1}^{\prime} \neq k_{2}^{\prime} \leq K^{\prime}} \\
& \left(\mid \text { Leaves }\left(\text { Child }\left(n_{p}, k_{1}\right)\right) \cap \text { Leaves }\left(\text { Child }\left(n_{p^{\prime}}, k_{1}^{\prime}\right)\right) \mid\right. \\
& \times \mid \text { Leaves }\left(\text { Child }\left(n_{p}, k_{2}\right)\right) \cap \text { Leaves }\left(\text { Child }\left(n_{p^{\prime}}, k_{2}^{\prime}\right)\right) \mid \\
& \left.\times \mid\left(\Sigma \backslash \text { Leaves }\left(n_{p}\right)\right) \cap\left(\Sigma \backslash \text { Leaves }\left(n_{p^{\prime}}\right)\right) \mid\right)
\end{aligned}
$$

In both trees, a ' $\succ$ '-type Split-Order relation satisfies the following conditions: $\sigma_{i_{1}}, \sigma_{i_{2}}$ are descendants of two different children of $n_{p}$ and also of two different children of $n_{p^{\prime}}$. In addition, $\sigma_{i_{3}}$ is not in either Leaves $\left(n_{p}\right)$ or Leaves $\left(n_{p^{\prime}}\right)$ (see Fig. 4). Assume that computing the size of a set intersection takes constant time (we will explain later how to compute it in constant time). Let the maximum number of children for a node be $d$. The computation of $\operatorname{Shared}_{\succ}\left(n_{p}, n_{p^{\prime}}\right)$ takes $O\left(d^{4}\right)$ time. 


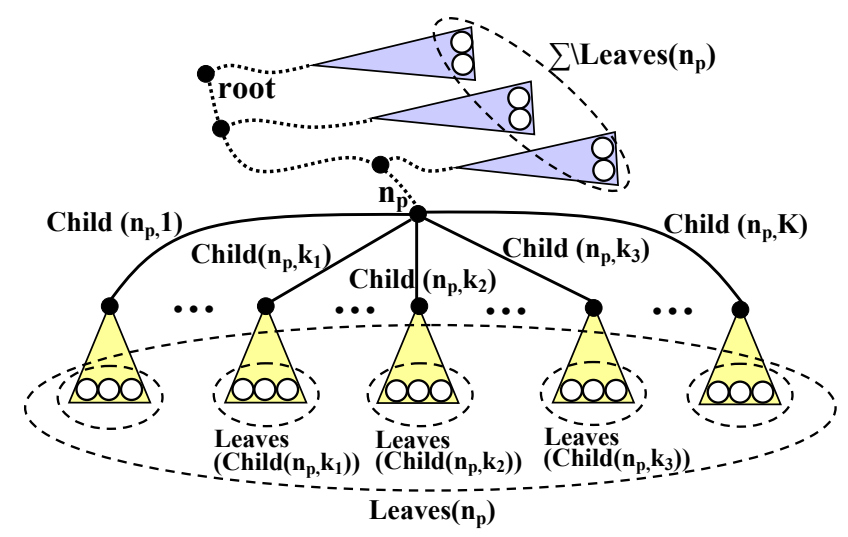

Fig. 4. Illustration of Split-Order distance computation.

The number of common '='-type Split-Order relations $\operatorname{Shared}_{=}\left(n_{p}, n_{p^{\prime}}\right)$ can be computed as follows:

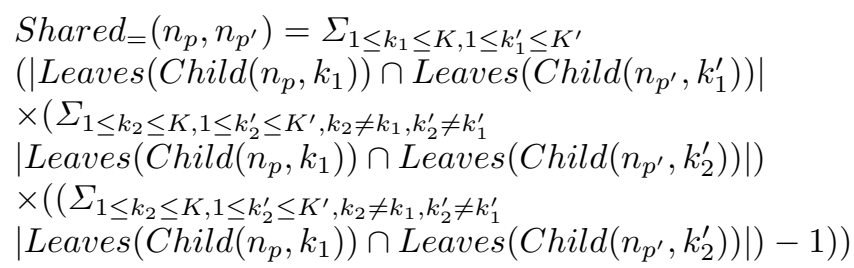

Similarly, in either tree, an '='-type Split-Order relation satisfies the following conditions: $\sigma_{i_{1}}, \sigma_{i_{2}}, \sigma_{i_{3}}$ are all descendants of $n_{p}$ and children of $n_{p}^{\prime}$; also, $\sigma_{i_{2}}$ and $\sigma_{i_{3}}$ must be descended from children which are not ancestors of $\sigma_{i_{1}}$; finally, $\sigma_{i_{2}}$ are $\sigma_{i_{3}}$ are different leaf labels (see Fig. 4). Again, if a set intersection computation takes constant time, the computation of Shared $_{=}\left(n_{p}, n_{p^{\prime}}\right)$ takes $O\left(d^{4}\right)$ time.

Therefore, let the total number of common ' $\succ$ '-type Split-Order relations shared between two trees be Shared $\succ\left(T, T^{\prime}\right)$, and the total number of common '='-type Split-Order relations shared between two trees be Shared $_{=}\left(T, T^{\prime}\right)$. Then we have:

$$
\begin{aligned}
& \operatorname{Shared}_{\succ}\left(T, T^{\prime}\right)=\Sigma_{\forall n_{p} \in T, n_{p^{\prime}} \in T^{\prime}} \operatorname{Shared}_{\succ}\left(n_{p}, n_{p^{\prime}}\right) \\
& \text { Shared }_{=}\left(T, T^{\prime}\right)=\Sigma_{\forall n_{p} \in T, n_{p^{\prime}} \in T^{\prime}} \text { Shared }_{=}\left(n_{p}, n_{p^{\prime}}\right)
\end{aligned}
$$

Thus, the total number of common Split-Order relations shared between $\operatorname{SplitOrderSet}\left(T, n_{p}\right)$ and $\operatorname{SplitOrderSet}\left(T^{\prime}, n_{p^{\prime}}\right)$ is:

$$
\operatorname{Shared}\left(T, T^{\prime}\right)=\text { Shared }_{=}\left(T, T^{\prime}\right)+2 \times \text { Shared }_{\succ}\left(T, T^{\prime}\right)
$$

Therefore, the Split-Order distance between $T$ and $T^{\prime}$ is:

$$
\operatorname{SODist}\left(T, T^{\prime}\right)=n^{3}-(n+3 n(n-1))-\operatorname{Shared}\left(T, T^{\prime}\right)
$$


Here $n^{3}$ is the total number of Split-Order relations for a leaf label set of size $n$, and $n+3 n(n-1)$ is the number of Split-Order relations of which at least two of $\sigma_{i_{1}}, \sigma_{i_{2}}, \sigma_{i_{3}}$ have the same label. As mentioned before, these Split-Order relations must be common for both trees.

Now we explain how we can compute the size of the set intersection in constant time. The basic idea is that we compute in advance $\mid$ Leaves $\left(n_{p}\right) \cap$ Leaves $\left(n_{p^{\prime}}\right) \mid$ for all possible pairs of $n_{p}$ and $n_{p^{\prime}}$, which are the internal nodes of $T$ and $T^{\prime}$, respectively. This can be done in $O\left(n^{2}\right)$ time as follows:

1. Initially, we compute $\mid$ Leaves $\left(n_{p}\right) \cap$ Leaves $\left(n_{p^{\prime}}\right) \mid$ where $n_{p}$ and $n_{p^{\prime}}$ are leaf nodes in $T$ and $T^{\prime}$, respectively. Each $\mid$ Leaves $\left(n_{p}\right) \cap$ Leaves $\left(n_{p^{\prime}}\right) \mid$ can be done in constant time.

2. We compute $\mid$ Leaves $\left(n_{p}\right) \cap$ Leaves $\left(n_{p^{\prime}}\right) \mid$ if either of the following cases holds: (a) $\mid$ Leaves $\left(\operatorname{Child}\left(n_{p}, k\right)\right) \cap$ Leaves $\left(n_{p^{\prime}}\right) \mid$ has been computed for all children of $n_{p}$

(b) $\mid$ Leaves $\left(n_{p}\right) \cap$ Leaves $\left(\operatorname{Child}\left(n_{p^{\prime}}, k^{\prime}\right)\right) \mid$ has been computed for all children of $n_{p^{\prime}}$

This can be done in $O(d)$ time where $d$ is the maximum degree of any node. For case (a):

$$
\begin{aligned}
& \mid \text { Leaves }\left(\operatorname{Child}\left(n_{p}, k\right)\right) \cap \operatorname{Leaves}\left(n_{p^{\prime}}\right) \mid= \\
& \Sigma_{k}\left|\operatorname{Leaves}\left(\operatorname{Child}\left(n_{p}, k\right)\right) \cap \operatorname{Leaves}\left(n_{p^{\prime}}\right)\right|
\end{aligned}
$$

For case (b):

$$
\begin{aligned}
& \mid \text { Leaves }\left(\operatorname{Child}\left(n_{p}, k\right)\right) \cap \text { Leaves }\left(n_{p^{\prime}}\right) \mid= \\
& \Sigma_{k^{\prime}} \mid \text { Leaves }\left(n_{p}\right) \cap \text { Leaves }\left(\operatorname{Child}\left(n_{p^{\prime}}, k^{\prime}\right)\right) \mid
\end{aligned}
$$

3. Repeat 2) until $\mid$ Leaves $\left(n_{p}\right) \cap$ Leaves $\left(n_{p^{\prime}}\right) \mid$ is computed for any pair of nodes $n_{p} \in T$, and $n_{p^{\prime}} \in T^{\prime}$

Therefore, computing Leaves $\left(n_{p}\right) \cap$ Leaves $\left(n_{p^{\prime}}\right)$ for all possible pairs of $n_{p}$ and $n_{p^{\prime}}$ takes $O\left(d n^{2}\right)$ time. Note that in the computation of $\operatorname{Shared}_{\succ}\left(n_{p}, n_{p^{\prime}}\right)$, we also need to compute $\mid\left(\Sigma \backslash\right.$ Leaves $\left.\left(n_{p}\right)\right) \cap\left(\Sigma \backslash\right.$ Leaves $\left.\left(n_{p^{\prime}}\right)\right) \mid$, which can be computed in constant time as follows:

$$
\begin{aligned}
& \mid\left(\Sigma \backslash \text { Leaves }\left(n_{p}\right)\right) \cap\left(\Sigma \backslash \text { Leaves }\left(n_{p^{\prime}}\right)\right) \mid= \\
& n-\mid \text { Leaves }\left(n_{p}\right)|-| \text { Leaves }\left(n_{p^{\prime}}\right) \mid+ \\
& \mid \text { Leaves }\left(n_{p}\right) \cap \text { Leaves }\left(n_{p^{\prime}}\right) \mid
\end{aligned}
$$

We can easily compute $\mid$ Leaves $\left(n_{p}\right) \mid$ and $\mid$ Leaves $\left(n_{p^{\prime}}\right) \mid$ in advance for all $n_{p} \in T$ and $n_{p^{\prime}} \in T^{\prime}$ using a post-order traversal for both trees, which takes $O(n d)$ for each tree.

The complete algorithm for computing Split-Order distance is in Algorithm 2.

Theorem 3. Algorithm 2 runs in $O\left(n^{2} d^{4}\right)$ time.

Proof. To compute SODist $\left(T, T^{\prime}\right)$, we consider every pair of nodes $\left(n_{p}, n_{p^{\prime}}\right)$. The number of internal nodes for both trees are $O(n)$, therefore, we have $O\left(n^{2}\right)$ node pairs. Since computing $\operatorname{Shared}\left(n_{p}, n_{p^{\prime}}\right)$ takes $O\left(d^{4}\right)$ time, the total time complexity of Algorithm 2 is $O\left(n^{2} d^{4}\right)$. 


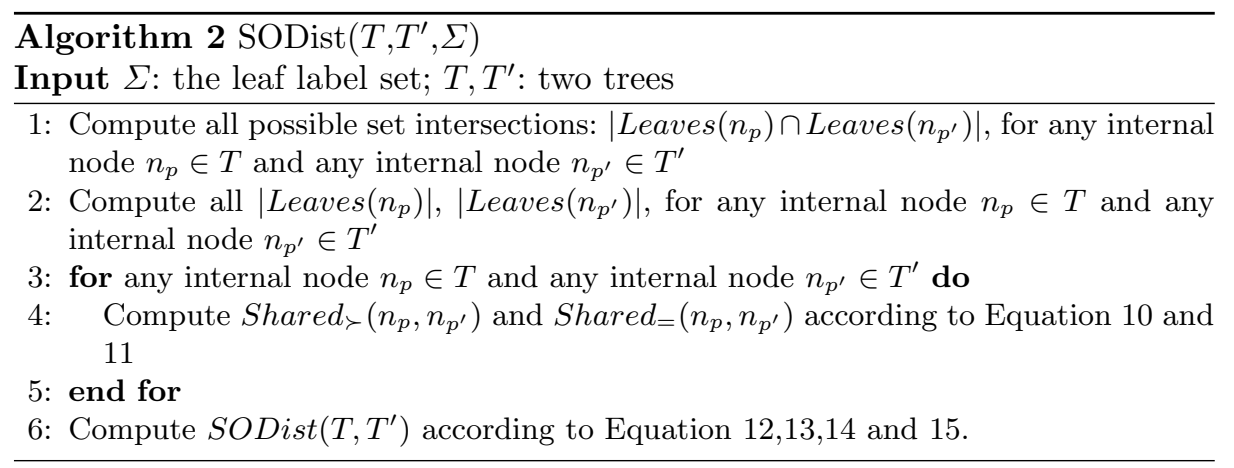

\section{$6 \quad$ Experimental Results}

We test the performance of our algorithm on both real and synthetic data sets.

- Iyer's Data[Iyer96]: Iyer's Data contains gene expression levels of 517 human genes in response to serum stimulation over 12 time points. The 517 genes can be clustered into hierarchical structures based on their coexpressions [Iyer96,Jiang03].

- Synthetic Data: 1) SYN-Random: The synthetic dataset contains a set of randomly generated, rooted, leaf labeled, unordered trees. The simulation is controlled by two parameters: the number of leaves $n$, and the maximum degree $d$ of any internal node. 2) SYN-2D: The 2-D synthetic dataset contains 1000 sampled pixels from 4 shapes in an image, including $1 \%$ of background noises. Clustering hierarchy for Syn-2D is obtained by applying CLUTO software ${ }^{1}$. The 4 clusters (representing the four big branches in the hierarchy) are illustrated using different colors in Fig. 6(a).

We compared the performance of our algorithm against the tree edit distance. We used a Java implementation of Zhang-Shasha's tree edit distance algorithm [Zhang89] available online ${ }^{2}$. Zhang-Shasha's algorithm applies to ordered, labeled trees. Our Split-Order algorithm is implemented in both $\mathrm{C}++$ and Java, and the experiments are performed on an Intel Core 2 Duo 1.6 GHz machine with $3 \mathrm{~GB}$ memory. The Java version of the Split-Order algorithm is mainly used for the comparison of running time performance with the Java implementation of Zhang-Shasha's tree edit distance algorithm.

\subsection{Distance Evaluation}

Distance Comparison on Iyer's Data Iyer's Data contains gene expression levels of 517 human genes over 12 time points. We refer to the complete Iyer's Data as Iyer12. Six additional data sets are generated from Iyer12 by randomly

\footnotetext{
${ }^{1}$ http://glaros.dtc.umn.edu/gkhome/views/cluto

${ }^{2}$ http://www.ics.mq.edu.au/ swan/howtos/treedistance/package.html
} 


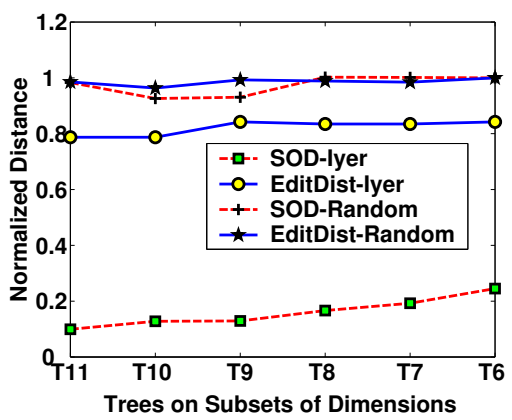

Fig. 5. Comparison of Split-Order distance and tree edit distance (Zhang-Shasha) on Iyer's data

choosing $11,10,9,8,7,6$ dimensions respectively. These data sets are referred to as Iyer11, Iyer10, Iyer9, Iyer8, Iyer7, and Iyer6. We apply the same hierarchical clustering algorithm on these 7 data sets with Euclidean distance and single linkage, and obtain 7 trees: $T_{12}, T_{11}, T_{10}, T_{9}, T_{8}, T_{7}$, and $T_{6}$. As we remove more and more dimensions, the correlation captured by the resulting tree differs more and more from the original tree $T_{12}$. We would therefore expect that the distances of $T_{11}, T_{10}, T_{9}, T_{8}, T_{7}$, and $T_{6}$ to $T_{12}$ are in an increasing order.

Fig.5 compares the distance scores computed using Split-Order distance and tree edit distance on the Iyer's Data. The Split-Order scores are normalized with the Split-Order distance of a random tree to $T_{12}$ which is 0.664 . Similarly, the tree edit distance scores are normalized with the tree edit distance between a random tree and $T_{12}$ which is 1433 . The Split-Order distance curve demonstrates a clear increasing trend for distances of $T_{11}, T_{10}, T_{9}, T_{8}, T_{7}$, and $T_{6}$ to the original tree $T_{12}$, which is consistent with our expectation. Compared to tree edit distance, Split-Order distance generates a much smaller distance for similar trees. The Split-Order distance between $T_{11}$ and $T_{12}$ is $10 \%$ of the distance between a random tree and $T_{12}$. The tree edit distance between $T_{11}$ and $T_{12}$ is however $80 \%$ of the distance between a random tree and $T_{12}$.

We also generated a random matrix of the same size (517 by 12) and performed the same experiment on this data. The corresponding curves are plotted in Fig.5. The two curves on random data are flat. The different behaviors of the random data curves and the Iyer's data curves can be explained by the gene correlation existing in the Iyer's data and the captured tree similarity due to the data correlation.

The average running time of Split-Order distance and tree edit distance in this experiment is $0.374 \mathrm{sec}$ and $120.2 \mathrm{sec}$ respectively.

Distance Evaluation on Synthetic Data We performed the distance evaluation on clustering hierarchies generated using SYN-2D. SYN-2D contains 1000 pixels uniformly sampled from 4 different shapes in an image, including $1 \%$ of background noise. We generated 5 additional data sets by randomly choosing 


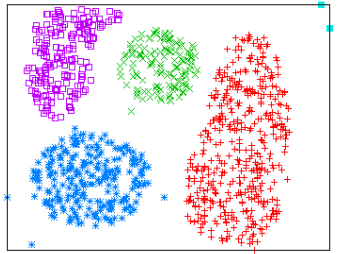

(a) $T_{0}$

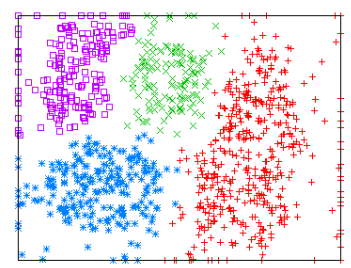

(d) $\operatorname{SODist}\left(T_{0}, T_{30}\right)=0.176$

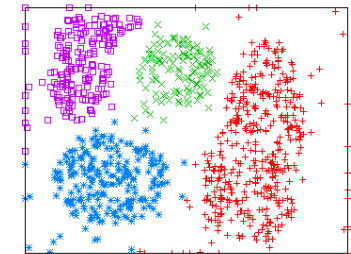

(b) $\operatorname{SODist}\left(T_{0}, T_{10}\right)=0.087$

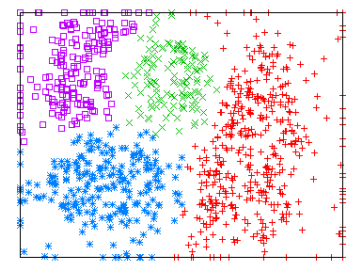

(e) $\operatorname{SODist}\left(T_{0}, T_{40}\right)=0.217$

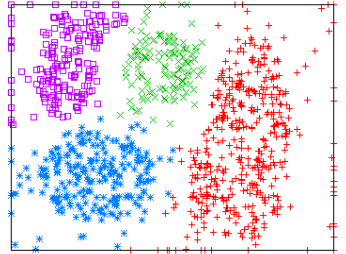

(c) $\operatorname{SODist}\left(T_{0}, T_{20}\right)=0.128$

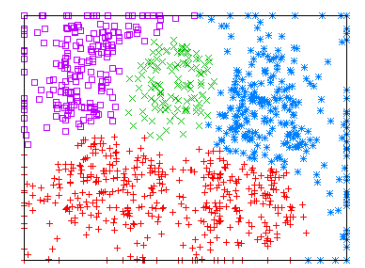

(f) $\operatorname{SODist}\left(T_{0}, T_{50}\right)=0.458$

Fig. 6. Illustration of 4 big clusters in the clustering hierarchies computed for SYN-2D and its perturbed data sets. $T_{0}$ is the clustering hierarchy computed on SYN-2D, $T_{10}$, $T_{20}, T_{30}, T_{40}, T_{50}$ are the clustering hierarchies computed on SYN-2D with $10 \%, 20 \%$, $30 \%, 40 \%, 50 \%$ of points applied perturbation.

$10 \%, 20 \%, 30 \%, 40 \%$, and $50 \%$ of pixels in SYN-2D and applyed a random perturbation on each pixel. Clustering hierarchies are generated on all 6 data sets using the hierarchical clustering function equipped with CLUTO [Karypis02]. We refer to the corresponding trees as $T_{0}, T_{10}, T_{20}, T_{30}, T_{40}$, and $T_{50}$. The four big clusters (the four big branches) in each tree are plotted in Fig.6(a)-Fig.6(f).

The Split-Order distances of each of $T_{0}$ to $T_{10}, T_{20}, T_{30}, T_{40}$, and $T_{50}$ are $0.087,0.128,0.176,0.216$, and 0.458 , respectively. The visual differences among Fig.6(a)-Fig.6(f) reflect the clustering hierarchy differences among the corresponding trees. The increasing order of the Split-Order differences are accordant with the increasing visual differences shown in Fig.6(b) to Fig.6(f). Particularly, from $T_{50}$ to $T_{40}$, the Split-Order distance increases dramatically by more than 100\%. Comparing Fig. 6(e) and Fig. 6(f), we also observe a big change: the blue cluster in $6(\mathrm{e})$ becomes part of the red cluster in $6(\mathrm{f})$; part of the red cluster in $6(\mathrm{e})$ becomes blue cluster in 6(f). This implies that the big branch representing the blue cluster in $T_{40}$ switches positions with a subbranch of the red cluster. This change impacts the relationship between a large number of pixels, which results in a big difference in the corresponding Split-Order distances.

\subsection{Running Time Performance}

We compare the running time performance of our algorithm (the Java version) with tree edit distance (Java implementation of Zhang-Shasha algorithm) on the SYN-Random dataset. 


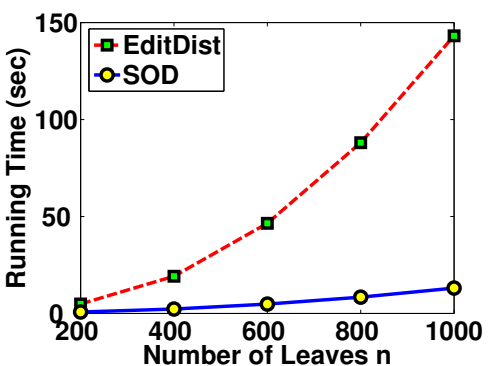

(a)

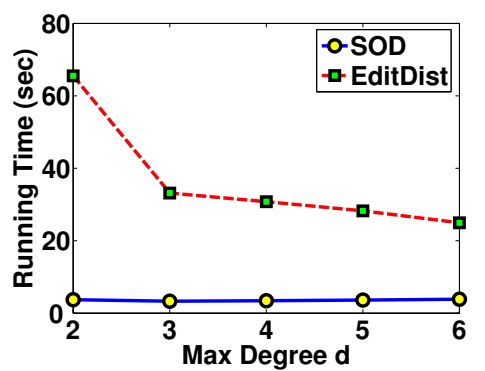

(b)

Fig. 7. Running time comparison of Split-Order distance (the Java implementation) and tree edit distance (Java implementation of Zhang-Shasha) with varying number of leaf nodes $n((\mathrm{a}))$ and with varying max degree $d((\mathrm{~b}))$.

Running time vs. $\boldsymbol{n}$ : Fig. 7(a) compares the running time of Split-Order and Zhang-Shasha (both implemented in Java) on trees with varying number of leaves $n$. As $n$ increases from 200 to 1000, the running time for both algorithms increases. The Split-Order distance is up to 10 times faster than the ZhangShasha algorithm and demonstrates better scalability with increasing $n$. Each data point is the average time taken for 10 distance computations for a given $n$. Although the running time of Zhang-Shasha is acceptable for reasonably large trees (less than 150 seconds for trees with 1000 leaves), the speedup would still be necessary considering large number of tree edit distance computations required for applications such as querying phylogeny tree databases.

Running time vs. $\boldsymbol{d}$ : Fig. 7(b) compares the running time of Split-Order and Zhang-Shasha (both implemented in Java) on trees with varying maximum degree $d$. The tree edit distance demonstrates a clear decline with increasing $d$, due to reduced total number of nodes with increasing $d$ and fixed number of leaves $n$. Split-Order remains almost flat with small variations. The time complexity of our algorithm depends on the number of internal nodes (which is $O(n))$ and the maximum degree of any node $d$. The running time would increase with larger $d$. However, with fixed $n$ and increasing $d$, the number of internal nodes decreases, which cancels out the increase introduced by larger $d$. The data is averaged over 10 runs of the algorithms.

\subsection{SODist Distribution}

We examined the distribution of SODist between any two trees using SYNRandom. 100 pairs of random trees are chosen and the relative Split-Order distances (SODistRel) are computed for each pair. Fig. 8(a) and 8(b) illustrate the distribution of the average SODistRel with varying parameters. We observe that the average SODistRel between any two random trees roughly falls 


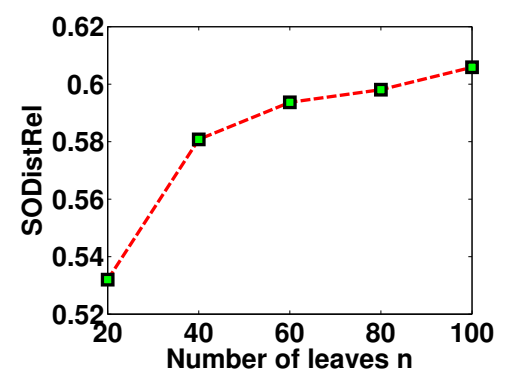

(a) Average SODistRel vs. $n$

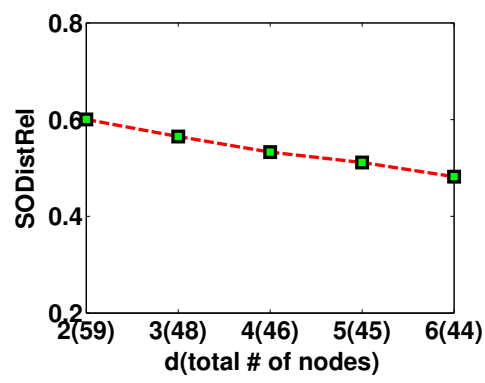

(b) Average SODistRel vs. d

Fig. 8. The average SODistRel between random trees with varying parameters: $n$ (the number of leaf nodes) and $d$ (the maximum number of children an internal node could have in a tree). $d$ is fixed to be 3 in (a) and $n$ is fixed to be 30 in (b).

within the range $[0.5,0.6]$. There are small variations when parameters change. As shown in Fig. 8(a), SODistRel ranges between 0.53 and 0.61 as $n$ varies from 20 to 100 and $d$ is fixed to be 3 . The increase in the average SODistRel between random trees is due to the increased topological variety of trees when $n$ is larger. In Fig. 8(b), the average SODistRel between random trees is computed as $d$ varies from 2 to 6 , and $n$ is fixed to be 30 . We can observe a slow drop in the average SODistRel between random trees when $d$ increases. This is due to the decrease in the number of internal nodes with increasing $d$ and fixed $n$. As tree becomes flatter, the amount of topological variety of trees also decreases.

Fig. 9(a) -9 (d) plots the histogram of 500 SODistRels between 500 pairs of random trees with different parameters settings: $n=20$ and $d=2$ (Fig. 9(a)), $n=20$ and $d=6$ (Fig. 9(b)), $n=100$ and $d=2$ (Fig. 9(c)), $n=100$ and $d=6$ (Fig. 9(d)). We observe that all distributions have the bell shape. For larger $d$ $(d=6$ compared to $d=2)$, more diversity exists in the tree space and histogram is more symmetric. For a larger $n(n=100$ compared to $n=20)$, SODistRel becomes larger, and the mean of the distribution moves further to the right. These histograms depict the variation of the tree space when parameters change evaluated by the Split-Order distance.

For comparison, we plot the four corresponding histograms of tree edit distances computed over the same 500 pairs of random trees in Figs. 9(e), 9(f),9(g) and $9(\mathrm{~h})$. The edit distance scores are normalized by $4 n$ to get the relative scores. $4 n$ is the upper bound of the tree edit distance between two trees with $n$ leaves. We observe that when $d$ increases (from 2 to 6 ), the variance of the tree edit distance declines. This contradicts the fact that the topological diversity increases as $d$ increases. This suggests that the tree edit distance is not an ideal measure. 


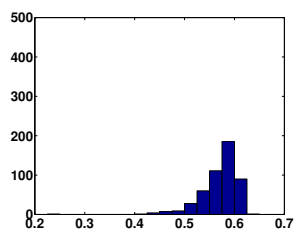

(a) $n=20, d=2$

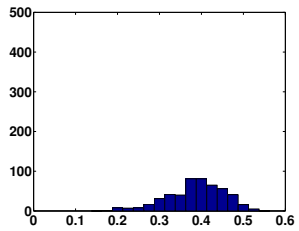

(e) $n=20, d=2$

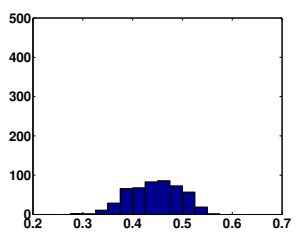

(b) $n=20, d=6$

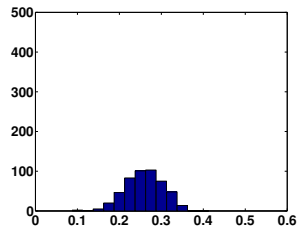

(f) $n=20, d=6$

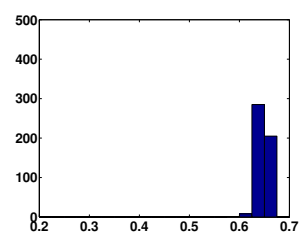

(c) $n=100, d=2$

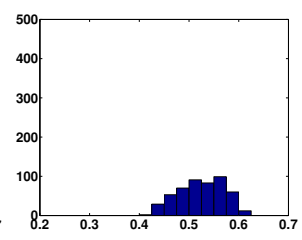

(d) $n=100, d=6$

Fig. 9. The distribution of SODistRel and tree edit distance between random trees in different settings. A total number of 500 Split-Order distance computations are performed. X axis is SODistRel or tree edit distance, $\mathrm{Y}$ axis is the number of the distance computations contained in each bin.

\section{Conclusion and Future Work}

In this paper, we propose a novel metric, Split-Order distance, to evaluate the distance between two clustering/classification hierarchies. This metric compares the order of the splits in both trees. We proved that a complete set of the split order relations uniquely determines the hierarchical structure. We also proposed an algorithm for reconstructing the tree using the set of order relations. Furthermore, we presented an efficient algorithm for computing the Split-Order distance between two hierarchies which takes $O\left(n^{2} d^{4}\right)$ time, where $n$ is the number of leaf nodes (objects), and $d$ is the maximum number of children in a tree.

In the future, we would like to take weighted edges into consideration where weights represent the distance from a node to its parent. This allows us to model the situations where a single edge with different weights can result in different relationships among objects, such as in a dendrogram.

\section{References}

[Allen01] Allen, B.L., Steel, M.: Subtree transfer operations and their induced metrics on evolutionary trees. Annals of Combinatorics, 5:1-13 (2001)

[Amir97] Amir, A., Keselman, D.: Maximum agreement subtree in a set of evolutionary trees: metrics and efficient algorithms. Proc. of the SIAM Journal on Computing, 26(6):1656-1669 (1997)

[Bille05] Bille, P.: A survey on tree edit distance and related problems. Theoretical Computer Science, 217-239 (2005) 
[Demaine07] Demaine, E.D., Mozes, S., Rossman, B., Weimann, O.: An Optimal Decomposition Algorithm for Tree Edit Distance. Proc. of the 34th International Colloquium on Automata, Languages and Programming (ICALP 2007)

[Estabrook85] Eastabrook, G.F., McMorris, F.R., Meacham, C.A.: Comparison of undirected phylogenetic trees based on subtrees of four evolutionary units. Syst. Zool. (1985)

[Felsenstein78] Felsenstein, J.: Cases in which parsimony and compatibility methods will be positively misleading. Syst. Zool., 27:401-410 (1978)

[Iyer96] Iyer V.R. et al.: Transcriptional program in the response of human fibroblasts to serum. Science, 283:83-87 (1996)

[Jiang03] Jiang, D., Pei, J., Zhang, A.: DHC: a density-based hierarchical clustering method for time series gene expression data. Proc. of the The Third Symposium on Bioinformatics and Bioengineering, 393-400 (2003)

[Karypis02] Karypis, G.: CLUTO - A Clustering Toolkit. Tech Report, Dept. of Computer Science, University of Minnesota, 2002

[Klein98] Klein, P.N.: Computing the edit-distance between unrooted ordered trees. Proc. of the 6th annual European Symposium on Algorithms (ESA) 1998

[Lu79] Lu, S.Y.: A tree-to-tree distance and its application to cluster analysis. IEEE Transactions on Pattern Analysis and Machine Intelligence (PAMI) 1979

[Robinson79] Robinson, D.F., Foulds, L.R.: Comparison of weighted labeled trees. Combinatorial mathematics, VI 119-126 (1979)

[Robinson81] Robinson, D.F., Foulds, L.: Comparison of phylogenetic trees. Math. Biosci., 53(1-2):131-147 (1981)

[Saitou87] Saitou, N., Nei, M.: The neighbor-joining method: a new method for reconstructing phylogenetic trees. The Mol. Biol. Evol., 4(4):406425 (1987)

[Shasha04] Shasha, D., Wang, J.T.L., Zhang, S.: Unordered Tree Mining with Applications to Phylogeny. Proc. IEEE International Conference on Data Engineering (ICDE'04)

[Sneath73] Sneath, P.H.A., Sokal, R.R.: Numerical Taxonomy. WH Freeman and Company, 230-234 (1973)

[Touzet03] Touzet, H.: Tree edit distance with gaps. Information Processing Letters, 85(3): 123-129 (2003)

[Wang94] Wang, J.T., Zhang, K., Jeong, K., Shasha, D.: A system for approximate tree matching. IEEE Transactions on Knowledge and Data Engineering, 6(4): 559$571(1994)$

[Wang03] Eastabrook, G.F., McMorris, F.R., Meacham, C.A.: TreeRank: A similarity measure for nearest neighbor searching in phylogenetic databases. Proc. of the 15th International Conference on Scientific and Statistical Database Management (1985)

[Waterman78] Waterman, M.S., Smith, T.F.: On the similarity of dendrograms. Journal of Theoretical Biology, 73:789-800 (1978)

[Zhang89] Zhang, K., Shasha, D.: Simple fast algorithms for the editing distance between trees and related problems. SIAM Journal of Computing (1989) 